Supplemental Digital Appendix 1

Discussion Guide, From a Study of Responses to Feedback Regarding Implicit Bias in Pediatric Health Care Professionals, Schulich School of Medicine and Dentistry, Western University, Ontario, 2015-2016

\title{
Discussion Questions
}

What is stigma?

What was it like to take the IAT [implicit association test]?

Was the experience positive or negative?

Were the results what you expected or not?

What do you think about the accuracy of the results?

What were your emotions while taking the test and receiving the result?

What were your cognitions (automatic thoughts, inner monologue) while taking the test and receiving the result?

If your results were different, would your experience change?

Knowing what you know now, do you believe your implicit attitudes can change?

What kind of change (attitudinal, behavioral, or both)? 